\title{
Comparison of plane mirror vs retroreflector performance for laser-self-mixing displacement sensors
}

\author{
S. Ottonelli \\ simona.ottonelli@fisica.uniba.it
}

\section{F. De Lucia}

\section{M. di Vietro}

\section{Dabbicco}

\section{G. Scamarcio}

CNR-INFM Regional Laboratory LIT3, Università degli Studi di Bari, Via Amendola 173, I-70126 Bari, Italy and Dipartimento Interateneo di Fisica "M. Merlin," Università degli Studi di Bari, Via Amendola 173, I-70126 Bari, Italy

CNR-INFM Regional Laboratory LIT3, Università degli Studi di Bari, Via Amendola 173, I-70126 Bari, Italy and Dipartimento Interateneo di Fisica "M. Merlin," Università degli Studi di Bari, Via Amendola 173, I-70126 Bari, Italy

CNR-INFM Regional Laboratory LIT3, Università degli Studi di Bari, Via Amendola 173, I-70126 Bari, Italy and Dipartimento Interateneo di Fisica "M. Merlin," Università degli Studi di Bari, Via Amendola 173, I-70126 Bari, Italy

CNR-INFM Regional Laboratory LIT3, Università degli Studi di Bari, Via Amendola 173, I-70126 Bari, Italy and Dipartimento Interateneo di Fisica "M. Merlin," Università degli Studi di Bari, Via Amendola 173, I-70126 Bari, Italy

CNR-INFM Regional Laboratory LIT3, Università degli Studi di Bari, Via Amendola 173, I-70126 Bari, Italy and Dipartimento Interateneo di Fisica "M. Merlin," Università degli Studi di Bari, Via Amendola 173, I-70126 Bari, Italy

The behavior of a displacement optical sensor based on the Laser-Self-Mixing effect employing a plane mirror (PT) and a solid corner-cube (CC) as moving target is analyzed. The performance of the sensor is compared in terms of simplification of the optical setup, measurement accuracy and tolerance to angular misalignment of the target. On the basis of the innovative assumption that only the fraction of the laser beam orthogonal to the target plane gives rise to the Self - Mixing modulation, it is demonstrated that the interferometer tolerates small tilt of the plane target (up to approximately $0.7^{\circ}$ ) only when illuminated by a divergent beam, in which case the displacement measurement becomes affected by a cosine - type error. Instead the corner cube preserves the self-mixing signal over a larger angular range (up to approximately $2^{\circ}$ ) at the same time preserving high measurement accuracy. [D0I: 10.2971/jeos.2009.09036]

Keywords: self-mixing, optical feedback, displacement measurement, corner-cube, target misalignment

\section{INTRODUCTION}

Laser interferometry is one of the most disseminate and performing phase sensitive techniques for metrological measurements. Besides the traditional "external configuration" needing one or more external detectors, in the last two decades a new kind of interferometry, called Laser-Self-Mixing (LSM) effect, has been established [1].

The self-mixing interference occurs inside the laser cavity between the standing wave inside the cavity of the laser and part of the laser beam re-entering the cavity after being coherently reflected or back-scattered by an external target. The result of the interaction between the two beams is a periodic change of the value of some laser emission parameters, such as the wavelength, the threshold current, the carrier density and the output power. The most suitable solution, in order to optimize the measuring setup, consists in the detection of the amplitude modulation of the laser output power by means of the photodiode integrated into the laser package for feedback control.

The useful features of this scheme with respect to the traditional interferometric one are the reduction of the number of required optical elements (such as the beam splitter and the reference mirror), a much simpler optical alignment since there is no external reference arm, and a reduced cost due to the absence of the external detector. Thanks to these benefits, the self-mixing has been recognized as a useful technique in a great variety of metrological applications [2], primarily for measuring displacement, velocity and distance.

Another peculiarity of this scheme regards the shape of the detected signal which is not simply sinusoidal, but depends on the feedback regimes, i.e. the relative amount of light coupled back into the laser. A useful classification of the feedback regimes for metrological purposes can be performed by adopting the $C$ - value as selective parameter, where $C$ is the feedback parameter [3] defined as follows:

$$
C=\varepsilon \sqrt{\frac{R_{3}}{R_{2}}}\left(1-R_{2}\right) \sqrt{1+\alpha^{2}} \frac{L}{l \cdot n_{e f f}}
$$

Eq. (1) depends on a combination of laser dependent parameters $\left(R_{2}\right.$ is the output coupler reflectivity, $l$ is the laser cavity length, $n_{e f f}$ is the effective refractive index of the active 
(a)

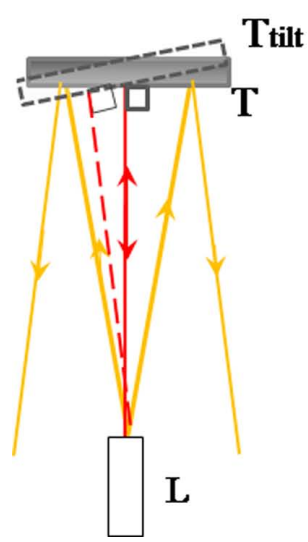

(b)

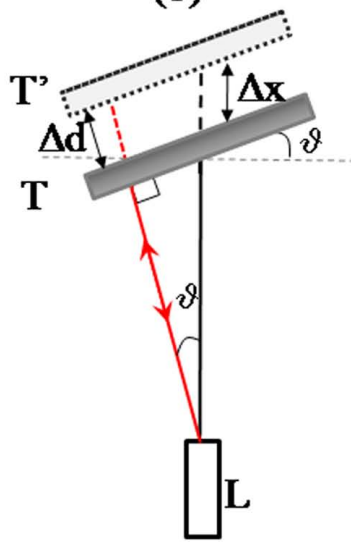

(c)

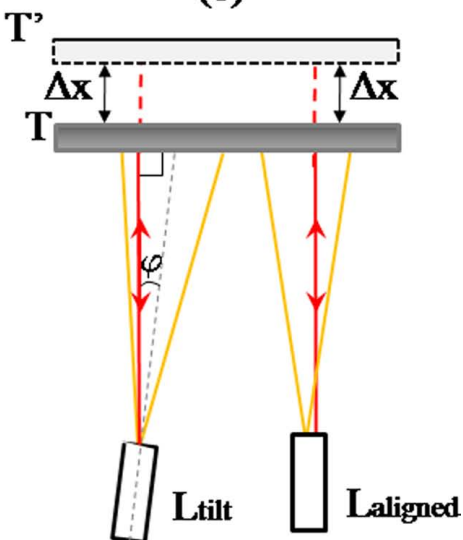

FIG. 1 (a) Misalignment of the target $T_{\text {tilt }}$ with respect to the optical axis. (b) Linear displacement $\Delta x$ in presence of a target tilted of an angle $\vartheta$. (c) Linear displacement $\Delta x$ measured by two lasers: $L_{\text {tilt }}$ misaligned of an angle $\varphi$ and $L_{\text {aligned }}$ well- aligned along the $x$ - axis The red line represents the fraction of the laser beam produced by the laser LD orthogonal to the target first $(T)$ and after $\left(T^{\prime}\right)$ the displacement.

medium, $\alpha$ is the linewidth enhancement factor) and system adjustable parameters $\left(R_{3}\right.$ is the target reflectivity, $L$ is the external cavity length, and $\varepsilon<1$ is a constant referred to as the mode matching factor).

By increasing the feedback power, the power modulation fringes appear sinusoidal $(C \ll 1$; very weak feedback regime), slightly asymmetric $(0.1<C<1$, weak feedback regime) or sawtooth-like $(1<C<4.6$, moderate feedback regime) with sharp switching every time the phase changes by $2 \pi$. The asymmetry of the output signal in the moderate feedback regime allows for an easy discrimination of the direction of motion by a single quadrature reading, on the basis of the sign of the fast slopes of the sawtooth-like signal. Thus, whereas the intrinsic $\lambda / 2$ displacement measurement resolution typical of interferometric setup can be retained by digital fringe count electronics, the discrimination of the direction of motion is recovered by the sign of the fast slopes of the sawtooth-like signal. By the AC derivation of the output signal and the algebraic sum $N=N_{+}-N_{-}$of the positive $\left(N_{+}\right)$/ negative $\left(N_{-}\right)$number of the peaks in the derivative, the linear displacements can be obtained as $\Delta X=N \times \lambda / 2$.

The target usually employed in a self - mixing interferometer, that is a single plane mirror (PT), has two drawbacks. First, the angular range of target misalignment is limited by the angular divergence of the laser beam. Second, a small tilt of the target results in an inclination of the plane - mirror with respect to the $x$ - axis, thus introducing a "cosine - type" error in the linear displacements along the $x$ - axis and limiting the measurement accuracy. Both these limitations can be overcome by using corner cubes (CC) in place of plane mirrors. In this paper, we compare the performance of a LSM interferometer for both kinds of targets and analyze the benefits of employing a corner-cube target.

\section{OPTIMAL COLLIMATION CONDITION AND MEASUREMENT ERRORS}

Operations in the moderate feedback regime, in a LSM apparatus with a plane mirror target, is usually achieved and controlled by means of a variable attenuator placed along the optical path. The attenuation of the feedback intensity is always required in presence of reflective target and collimated semiconductor lasers. In order to avoid the insertion of the filter, either the target should be changed or the collimating condition can be properly modified. In the first case, a diffusive target can be employed to reduce the back - scattered radiation re - entering the laser source; however, this solution implies a signal fading over long-distances due to the speckle effect. The alternative choice is represented by the reduction of the distance between the laser diode and collimating lens, in order to achieve a divergent beam and to obtain the following advantages:

1. no attenuation filter is required along the measuring arm.

2. the linear increase of the feedback parameter $C$ with the target distance can be tailored by a careful choice of the distance laser - target.

3. since only the fraction of the laser beam orthogonal to the target is back-reflected toward the lens following the same optical path, a divergent laser beam will accept larger target misalignment than a perfectly collimated laser beam. This principle is schematically illustrated in Figure 1(a), where the maximum allowed beam divergence will be given by the minimum feedback power ratio required to preserve the moderate - feedback regime.

The tolerance to angular misalignment described in point 3 has a two-fold implication: first, it allows a simpler optical alignment procedure than that required with collimated beams; second, it can be exploited for measuring yaw and pitch rotations of the target [4].

In spite of these benefits, an angular misalignment $\vartheta$ of the normal to the target with respect to the measurement axis, as- 


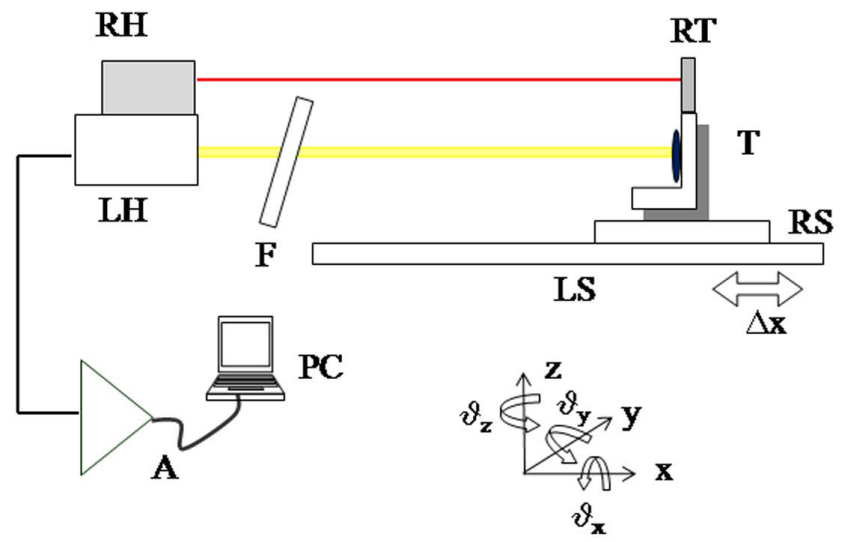

FIG. 2 Schematics of the sensor system: LH laser head, RH reference API head, T target, RT reference API target, LS linear stage, RS rotational stage, F variable filter, A trans- $Z$ amplifier, PC personal computer. The red (yellow) lines refer to the laser beam of the reference (proposed) system.

sumed as the $x$ - axis, affects the measurement of linear displacement. With reference to Figure 1(b), the LSM interferometer will measure a displacement $\Delta d$ along the direction orthogonal to the target, in place of the nominal displacement $\Delta x$ along the $x-$ axis. Since $\Delta d=\Delta x \cos (\vartheta)$, the relative error, given by the normalized difference between the measured displacement $\Delta d$ and the expected displacement $\Delta x$, is $\sigma / \Delta x=\cos (\vartheta)-1$. On the other hand, any misalignment $\varphi$ of the laser diode $L_{\text {tilt }}$ with respect to the $x$-axis (Figure 1(c)) will not bring in additional measurement errors, provided $\varphi$ to be less than the useful laser divergence. If we consider a maximum linear displacement $\Delta x=60 \mathrm{~cm}$, the absolute error is $\sigma=2 \mu \mathrm{m}$ for $\vartheta=0.1^{\circ}$ and $\sigma=91 \mu \mathrm{m}$ for $\vartheta=1^{\circ}$.

From the above considerations, it can be concluded that the measurement of linear displacement in a LSM interferometer employing a plane reflective target is affected by a cosine type measurement error due to angular misalignment of the target. This error is similar to the well - known cosine error affecting classical interferometric measurements, although the former is due to a misalignment of the target whereas the latter is caused by a misalignment of the laser diode, both referred to the direction $x$ of the linear motion.

The requirement of a divergent laser beam for measuring the target tilt angle can be relaxed by using a CC in place of the $\mathrm{PT}$, since in this case the backward optical path will not be affected by the target orientation. However, the cosine - type error affecting the measurement of linear displacement will again be related to misalignment of the laser as in classical interferometers.

\section{EXPERIMENTAL SETUP}

Figure 2 schematically illustrates the proposed sensor, composed of a laser head and a reflective target. The laser head is made up of a Distributed-Feedback (DFB) laser diode with nominal wavelength of $1310 \mathrm{~nm}$, current threshold $I_{t h}=$ $12 \mathrm{~mA}$ and biased at twice the threshold. It is equipped with monitor photodiode and collimated lens integrated into a single collimation tube. The current signal from the photodiode is firstly AC-coupled to a trans-impedance amplifier with gain of $10^{5} \mathrm{~V} / \mathrm{A}$ and then fed into a signal processing board interfaced to a computer. The moving target is made up by a squared plane mirror (PT) of side $5 \mathrm{~cm}$ or a retroreflector prism (CC) with a diameter of $10 \mathrm{~mm}$, rigidly fixed onto a rotation stage, mounted onto a 0.6-meter long linear stage. The minimum distance between the target and the laser head was $15 \mathrm{~cm}$. A commercial 6-axis measurement system (API $6 \mathrm{D}$ Laser), whose nominal resolution is $0.02 \mu \mathrm{m}$ for linear displacements, has been used as the reference meter.

\section{RESULTS AND DISCUSSION}

For validating the feasibility of this system as displacement sensor by using both PT and CC target, the linear stage was moved from a fixed initial position in the range $\pm 600 \mathrm{~mm}$ at a speed of $10 \mathrm{~mm} / \mathrm{s}$. The measurements were performed after a preliminary optical alignment of both the sources and the targets along the measurement $x$-axis. The focusing conditions were fixed in the collimation for the $\mathrm{CC}$, thus requiring a filter along the optical path to avoid instabilities due to strong feedback, and divergence of an angle $\alpha \approx 0.4^{\circ}$ for the PT.

Results reported in Figure 3 demonstrate that both PT and CC achieve a measurement accuracy $\sigma_{x}= \pm 20 \mu \mathrm{m}$ over $600 \mathrm{~mm}$ of linear displacement $\Delta x$. The linearity over the entire measurement range validates a useful dynamic range of at least five - orders of magnitude with the use of both kind of targets. The longest continuous measurable displacement was only limited by the length of our linear stage. However, we verified the existence of the sawtooth - like signal over a continuous range of $1.7 \mathrm{~m}$, with no adjustment required to the optics (the shortest distance from laser head was $0.15 \mathrm{~m}$ ).

To test the robustness of the proposed system against target misalignment, the laser beam was made increasingly more divergent by slight adjustments of the distance between the laser diode and the lens. For each divergence angle $\alpha$, the maximum angular misalignment $\beta$ of the target was determined as the greatest angle able to preserve a moderate feedback regime during a linear displacement. The results are shown in Figure 4, where the increasingly monotone trend confirms a greater tolerance to target misalignment up to approximately $0.7^{\circ}$, above which the feedback power was too low for the LSM signal to reach the moderate feedback regime.

On the other hand, the CC requires a small sized collimated beam, in order to reduce the phase distortions induced by the reflection at the faceted prism, and a filter attenuator since the CC reflects back almost $90 \%$ of the incident power. In Figure 5, the normalized number of counted fringes $N_{\text {norm }}$, obtained for a fixed linear displacements $\Delta x=600 \mathrm{~mm}$ in the case of a tilted target (along the $z$-axis), is reported for both kind of targets; the plane mirror was used with a divergent laser beam $\left(\alpha \approx(0.4 \pm 0.1)^{\circ}\right)$ with no filter, whereas the corner cube was used with a collimated laser beam and an optical filter. Starting from the zero position (with the front surface of the target orthogonal to the direction $x$ of linear motion), it can be observed a decreasing number of counted fringes for the plane 

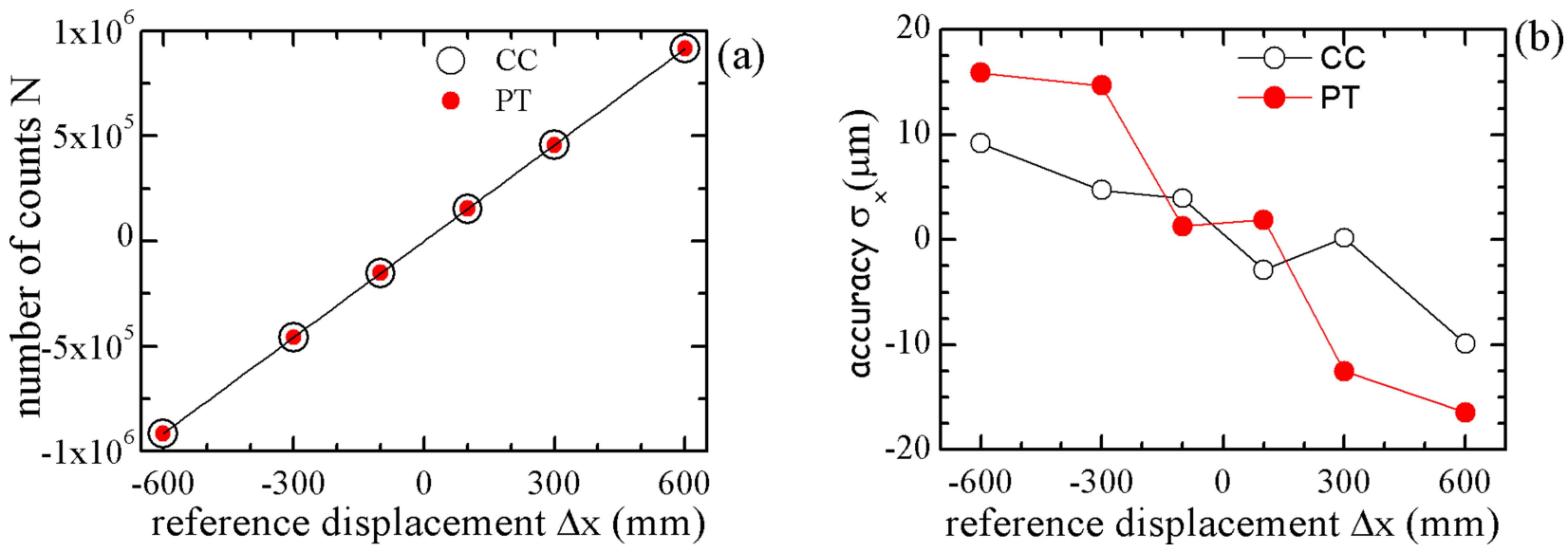

FIG. 3 Number $N$ of counted fringes (a) and measurement error $\sigma_{x}$ (b) for increasing linear displacements, as a function of the actual displacement measured by the reference system. Positive (negative) $\Delta x$ values indicate backward (forward) displacements.

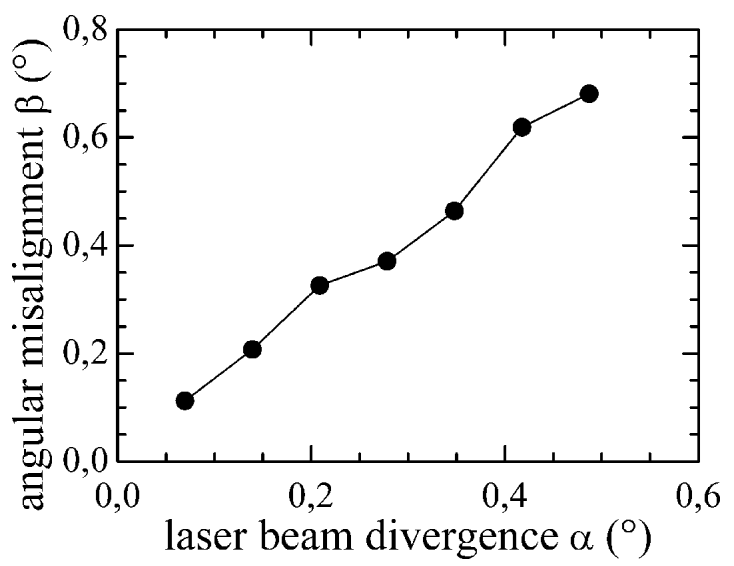

FIG. 4 Maximum tolerated misalignment $\beta$ of the plane target for increasing laser beam divergences $\alpha$.

mirror, consistent with the cosine - type error introduced by the tilt of the plane mirror.

Conversely, the corner cube illuminated by a collimated beam does not suffer of the same limitation. It tolerates a much larger target rotation up to some degrees, at the same time preserving the number of counted fringes within the experimental errors. This latter is mainly due to the cosine error induced by a slight misalignment of the laser source (angle $\varphi$ in Figure 1(c)) and wavelength instabilities of the laser source.

\section{CONCLUSION}

We compared the performance of a LSM sensor for measurement of linear displacements with two kinds of reflective targets, a plane mirror and a solid corner cube, in terms of simplification of the optical setup, measurement accuracy and tolerance to angular misalignment.

Two alternative solutions have been proposed for the improvement of the robustness of the optical apparatus against angular target misalignment: the use of a plane target with a diverging laser beam and the use of a corner cube target with a collimated laser beam. On the one hand, the plane mirror allows for the removal of the variable attenuator along

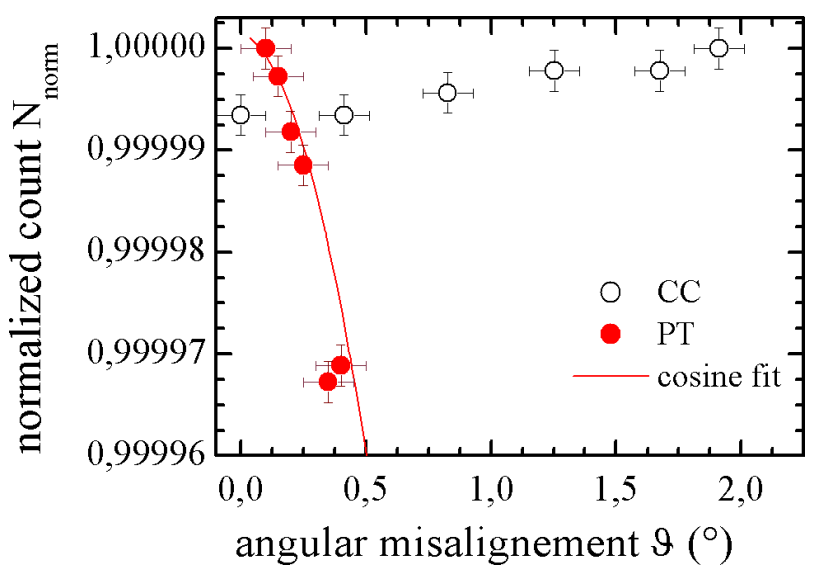

FIG. 5 Normalized number of counts $N_{\text {norm }}$ for a fixed linear displacement $\Delta x=600$ $\mathrm{mm}$, as a function of the tilt angle of the target around the vertical axis.

the optical path and causes the laser to tolerate small angular misalignment of the target within its angular divergence. The maximum demonstrated tilt angle of the target was approximately $0.7^{\circ}$. On the other hand, the use of a retroreflective target requires the insertion of the attenuator to avoid instabilities caused by strong feedback power, but makes the system completely insensitive to target misalignment up to at least $2^{\circ}$.

The full understanding of the different behavior of the system required a redefinition of what is usually referred to as the cosine-error. In case of plane mirror, the cosine - error is due to the target misalignment with respect to the measurement axis, whereas a fine alignment of the laser source along the longitudinal axis is not required since only the fraction of the laser beam orthogonal to the target contributes to the LSM effect. In case of retroreflective target the cosine-error retrieves the usual interpretation, as caused by a misalignment between the laser source and the measurement axis.

\section{References}

[1] S. Donati, Electro-Optical Instrumentation Chapter 4 (Prentice Hall, Upper Saddle River, NJ, 2004).

[2] G. Giuliani, M. Norgia, S. Donati, and T. Bosch, "Laser diode selfmixing technique for sensing applications" J. Opt. A: Pure Appl. Opt. 4, S283-\$294 (2002). 
[3] G. A. Acket, D. Lenstra, J. Den Boef, B. H. Verbeek, "The influence of feedback intensity on longitudinal mode properties and optical noise in index-guided semiconductor lasers" IEEE J. Quantum Electron. 20, 1163-1169 (1984).
[4] S. Ottonelli, F. De Lucia, M. di Vietro, M. Dabbicco, G. Scamarcio, and F. P. Mezzapesa, "A Compact Three Degrees-of-Freedom Motion Sensor Based on the Laser-Self-Mixing Effect" IEEE Phot. Technol. Lett. 20, 1360-1362 (2008). 